\begin{tabular}{|c|l|}
\hline Title & $\begin{array}{l}\text { UItrafast dynamics of Mott-state quench and formation in strongly correl ated BEDT-TTF molecular conductors } \\
\text { observed by three pul se pump probe spectroscopy }\end{array}$ \\
\hline Author(s) & Tsuchiya, S.; Taniguchi, H.; Y amada, J.; Toda, Y.; Mihail lovic, D.; Mertelj, T. \\
\hline Citation & $\begin{array}{l}\text { Physical Review B, 104(11), 115152 } \\
\text { https://doi.org/10.1103/PhysRevB.104.115152 }\end{array}$ \\
\hline Issue Date & 2021-09-24 \\
\hline Doc URL & http://hdl.handle.net/2115/83250 \\
\hline Rights & Copyright (2021) by The A merican Physical Society. \\
\hline Type & article \\
\hline File Information & PhysRevB.104.115152.pdf \\
\hline
\end{tabular}

Instructions for use 


\title{
Ultrafast dynamics of Mott-state quench and formation in strongly correlated BEDT-TTF molecular conductors observed by three-pulse pump probe spectroscopy
}

\author{
S. Tsuchiya, ${ }^{1,2}$ H. Taniguchi $\odot,{ }^{3}$ J. Yamada $\odot,{ }^{4}$ Y. Toda, ${ }^{1}$ D. Mihailovic,,${ }^{2,5}$ and T. Mertelj ${ }^{2,5}$ \\ ${ }^{1}$ Department of Applied Physics, Hokkaido University, Sapporo, Hokkaido 060-8628, Japan \\ ${ }^{2}$ Complex Matter Department, Jozef Stefan Institute, Jamova 39, SI-1000 Ljubljana, Slovenia \\ ${ }^{3}$ Graduate School of Science and Engineering, Saitama University, Saitama, 338-8570, Japan \\ ${ }^{4}$ Graduate School of Material Science, University of Hyogo, Hyogo 678-1205, Japan \\ ${ }^{5}$ Center of Excellence on Nanoscience and Nanotechnology Nanocenter, Jamova 39, SI-1000 Ljubljana, Slovenia
}

(Received 1 July 2021; revised 15 September 2021; accepted 16 September 2021; published 24 September 2021)

We investigate the quench and real-time formation of the Mott state and photoexcited carrier relaxation dynamics in the Mott insulator $\kappa$-(BEDT-TTF $)_{2} \mathrm{Cu}\left[\mathrm{N}(\mathrm{CN})_{2}\right] \mathrm{Cl}(\kappa-\mathrm{Cl})$ and the superconductor $\kappa$-(BEDT-TTF $)_{2} \mathrm{Cu}\left[\mathrm{N}(\mathrm{CN})_{2}\right] \mathrm{Br}(\kappa-\mathrm{Br})$ using three-pulse femtosecond optical spectroscopy, where BEDT-TTF is bis(ethylenedithio)tetrathiafulvalene. In both salts, we find that transient reflectivity amplitude gradually recovers with time after strong near-infrared pulse quench, but its relaxation time is nearly constant throughout. This indicates that in $\kappa-\mathrm{Cl}$, the energy gap for charge excitations is filled rather than closed by photoinduced carriers of only $\sim 0.5 \%$ per dimer. The Mott state is re-formed on a few-picosecond timescale with the disappearance of the in-gap photodoping-induced states near the Fermi energy. In $\kappa-\mathrm{Br}$, a behavior similar to that in $\kappa-\mathrm{Cl}$ is observed and attributed to the disorder-induced phase-separated Mott insulating regions.

DOI: 10.1103/PhysRevB.104.115152

\section{INTRODUCTION}

Metal-insulator transition in strongly correlated electron systems, more specifically the Mott-Hubbard transition, is one of the hottest topics in condensed-matter physics [1]. In cuprate superconductors, carrier doping induces the insulatormetal Mott-Hubbard transition, which is considered to be the key to understanding high-temperature superconductivity [2]. On the other hand, in organic superconductors, the Mott-Hubbard transition has been realized by modulation of effective correlation induced by atomic substitution and external pressure regardless of carrier doping [3]. Furthermore, in organic field-effect transistor devices, the Mott-Hubbard transition can be induced by controlling the gate voltage [4,5]. However, studies of the Mott-Hubbard transition have been largely limited to quasiequilibrium conditions.

A pump probe measurement with femtosecond laser pulses is often adopted to investigate low-energy electronic structure in an electronic ordered state and explore metastable states, which are not thermally accessible. The three-pulse pump probe spectroscopy, which is developed from ordinary pump probe spectroscopy, allows us to investigate the temporal evolution of the electronic state after the intense pulse irradiation in terms of carrier relaxation dynamics [6]. So far, this measurement has been applied to electronic ordered states characterized by spontaneous symmetry breaking and has revealed the growth of electronic order [7-9] and the dynamics of topological defects under nonequilibrium conditions [10], which are analogous to nonequilibrium phase transitions in cosmology [11,12].
The series of organic charge transfer salts $\kappa$-(BEDT$\mathrm{TTF})_{2} \mathrm{Cu}\left[\mathrm{N}(\mathrm{CN})_{2}\right] X(X=\mathrm{Cl}$ and $\mathrm{Br})$ is one of the most studied strongly correlated electron systems due to its rich electronic phases [13], where BEDT-TTF is bis(ethylenedithio)tetrathiafulvalene. In the Mott insulator $\kappa$ $\mathrm{Cl}(X=\mathrm{Cl})$, where the Mott gap was estimated to be $\sim 0.1 \mathrm{eV}$ [14-16], recent pump probe measurements have revealed that the photoinduced carrier dynamics of the Mott state becomes prominent below $\sim 80 \mathrm{~K}$ [17]. Moreover, in the superconductor $\kappa-\mathrm{Br}(X=\mathrm{Br})$, similar dynamics have been observed, indicating that the Mott state is locally induced by photoinduced modulation of the effective electron correlation [18]. Therefore, by applying three-pulse pump probe measurements to this system, we can investigate the temporal evolution of the Mott-state formation and the mechanism of the Mott-Hubbard transition under nonequilibrium conditions, which are never observed by ordinary quasiequilibrium experiments.

In this paper, we investigate the growth dynamics of the Mott state in $\kappa-\mathrm{Cl}$ and the photoinduced Mott state in $\kappa$ $\mathrm{Br}$ [19] under nonequilibrium conditions after irradiation by intense optical pulses at $1.53 \mathrm{eV}$ photon energy. The formation of the Mott state is indicated by the transient reflectivity dynamics in which the amplitude changes gradually with time while the relaxation time remains time independent in both salts. In $\kappa-\mathrm{Cl}$, the quench of the Mott state is realized by the generation of the in-gap states in combination with a chemical potential shift induced by a carrier excitation density of only $\gtrsim 0.5 \%$ per dimer. The recovery time of the Mott state is estimated to be $\sim 2$ ps and is similar in both the genuine Mott state in $\kappa-\mathrm{Cl}$ and the photoinduced phase-separated Mott state 
(a)

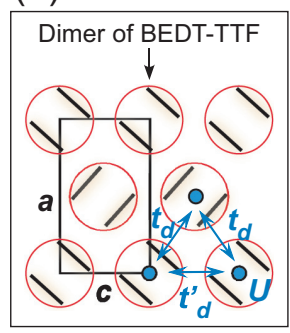

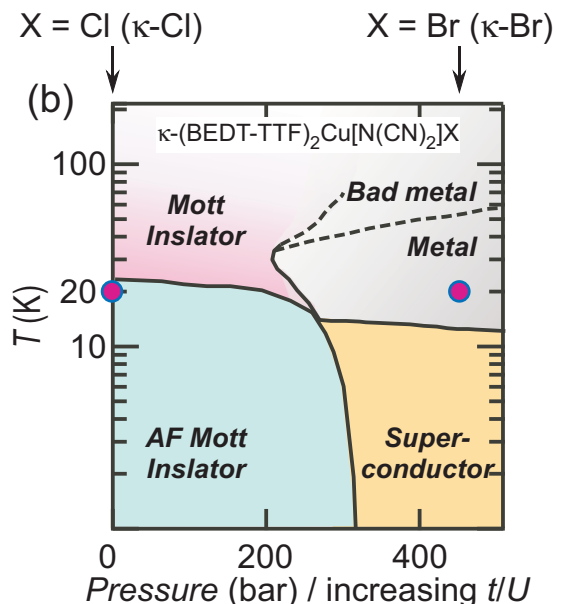

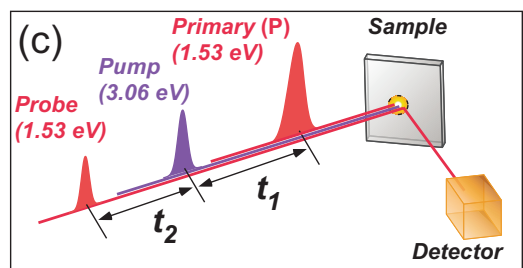

FIG. 1. (a) A schematic illustration of the BEDT-TTF molecular arrangement of a conducting layer. $U$ and $t_{d}$ and $t_{d}^{\prime}$ denote the onsite Coulomb repulsion and the transfer integrals between dimers, respectively. (b) Phase diagram of $\kappa$-(BEDT-TTF $)_{2} \mathrm{Cu}\left[\mathrm{N}(\mathrm{CN})_{2}\right] X$ $(X=\mathrm{Cl}$ and $\mathrm{Br})$. AF stands for antiferromagnetic. The solid circles correspond to a temperature of $20 \mathrm{~K}$, at which the measurements were conducted. (c) Schematic illustration of the three-pulse pump probe measurement. $t_{1}$ and $t_{2}$ denote delays between primary $(\mathrm{P})$ and pump pulses and pump and probe pulses, respectively.

in $\kappa$-Br. The results suggest that the formation of the Mott state is dominated by local correlations.

\section{EXPERIMENT}

Single crystals of $\kappa-\mathrm{Cl}$ and $\kappa-\mathrm{Br}$ were synthesized electrochemically $[20,21]$. Both salts have the same crystal structure, where BEDT-TTF and $\mathrm{Cu}\left[\mathrm{N}(\mathrm{CN})_{2}\right] X$ molecules form conducting and insulating layers, respectively, and they are alternately stacked along the $b$ axis. The atomic substitution from $X=\mathrm{Cl}$ to $\mathrm{Br}$ leads to a change in the effective electron correlation $t / U$ in the same way as the application of pressure, where $t$ symbolizes the transfer integral between the dimers of BEDT-TTF molecules $t_{d}$ and $t_{d}^{\prime}$ and $U$ is the on-site Coulomb repulsion, as shown in Fig. 1(a). Figure 1(b) shows the phase diagram. When $\mathrm{Cl}$ is substituted by $\mathrm{Br}$, the ground state is changed from the Mott insulator to superconductor with a transition temperature $T_{\mathrm{c}}$ of $\sim 12 \mathrm{~K}$ [13].

For the optical measurements, 50 fs pulses were obtained from a Ti: $\mathrm{Al}_{2} \mathrm{O}_{3}$ regenerative amplifier seeded with a Ti: $\mathrm{Al}_{2} \mathrm{O}_{3}$ oscillator. The pulse repetition rate was set to $100 \mathrm{kHz}$ to avoid the heating effect. To perform the threepulse pump probe measurements, pulses at $1.53 \mathrm{eV}$ photon energy were used for primary $(\mathrm{P})$ and probe pulses. Pulses at $3.06 \mathrm{eV}$ photon energy, which were obtained with standard frequency doubling in a $\beta-\mathrm{BaB}_{2} \mathrm{O}_{4}$ (BBO) crystal, were used for the pump pulse. According to the calculated band

structure [22], the energy of primary and probe pulses $(1.53 \mathrm{eV})$ almost corresponds to the next HOMO-HOMO transition, where next HOMO and HOMO denote the next highest occupied molecular orbital and the highest occupied molecular orbital, respectively. The energy of the pump pulse $(3.06 \mathrm{eV})$ is close to the optical transitions from the HOMO to bands well above the lowest unoccupied molecular orbital (LUMO) or from bands well below the HOMO to the LUMO.

Figure 1(c) shows a schematic of the three-pulse measurement. Three beams were focused almost perpendicularly to the conducting $(a c)$ plane, and the transient reflectivity $\Delta R / R$ was measured by the pump probe pulse sequence. The fluence of the P pulse was varied in the range of $43-231 \mu \mathrm{J} / \mathrm{cm}^{2}$, and those of the pump and probe pulses were fixed to 4 and $2 \mu \mathrm{J} / \mathrm{cm}^{2}$, respectively. The samples were carefully cooled in a helium flow cryostat at a rate of $1 \mathrm{~K} / \mathrm{min}$ to avoid the structural disorder of BEDT-TTF molecules [23]. The experiments were conducted at $20 \mathrm{~K}$, where the transient reflectivity dynamics due to the gap/pseudogap were observed to be dominant in both salts. In $\kappa-\mathrm{Cl}$, the gap arises from the Mott insulating state $[17,19]$. In the metallic state of $\kappa-\mathrm{Br}$, the pseudogap (PG) is interpreted as the generation of the Mott state in some parts of the sample by photoinduced modulation of $t / U[18,19]$. Since the measured temperature is higher than $T_{\mathrm{c}}$ in $\kappa-\mathrm{Br}$, there is no superconducting contribution to the transient reflectivity dynamics, and hence, very similar dynamics are observed for $\kappa-\mathrm{Cl}$ and $\kappa-\mathrm{Br}$. The probe polarization was fixed along the $c$ axis, where the gap-/PG-related transient reflectivity is the most enhanced $[17,18]$. The polarizations of the $\mathrm{P}$ and pump pulses were fixed along the $a$ axis, perpendicular to the probe polarization.

In the standard pump probe experiments, the photoexcited (PE) carriers in the nonequilibrium high-energy states by the pump pulse relax on an $\sim 100 \mathrm{fs}$ timescale to the states near the Fermi energy via electron-electron and electron phonon scattering, forming nonequilibrium phonon and carrier distributions [24]. The transition probability for the probe pulse involves absorptions, which can be given by Fermi's golden rule, reflecting the transition between the PE carrier state near the Fermi energy and the unoccupied state above the probe energy $(1.53 \mathrm{eV})$, as well as the transition between the unoccupied state near the Fermi energy and the occupied state below the probe energy. The transient change in reflectivity $\Delta R / R$ is written as

$$
\frac{\Delta R}{R}=\frac{R_{\text {pump }}-R_{0}}{R_{0}} \propto \Delta f N\left|M_{i j}\right|^{2},
$$

where $R_{\text {pump }}$ and $R_{0}$ are the reflectivities with and without pump pulse irradiation and $\Delta f, N$, and $M_{i j}$ are the distribution function of PE carriers, the density of electronic states, and the dipole matrix element, respectively. Through measurements of $\Delta R / R$ as a function of a delay of $t_{2}$ as shown in Fig. 1(c), the temporal evolution of $\Delta f$ can be investigated. If an energy gap for electronic excitations, such as a Mott insulating gap, forms, a relaxation bottleneck may occur $[25,26]$, and the dynamics drastically changes compared to the normal metallic state. Thus, we can extract information about the carrier dynamics and the nature of the gap at the Fermi energy.

In the three-pulse measurements an intense $\mathrm{P}$ pulse is applied before the pump and probe pulses arrive, as shown 

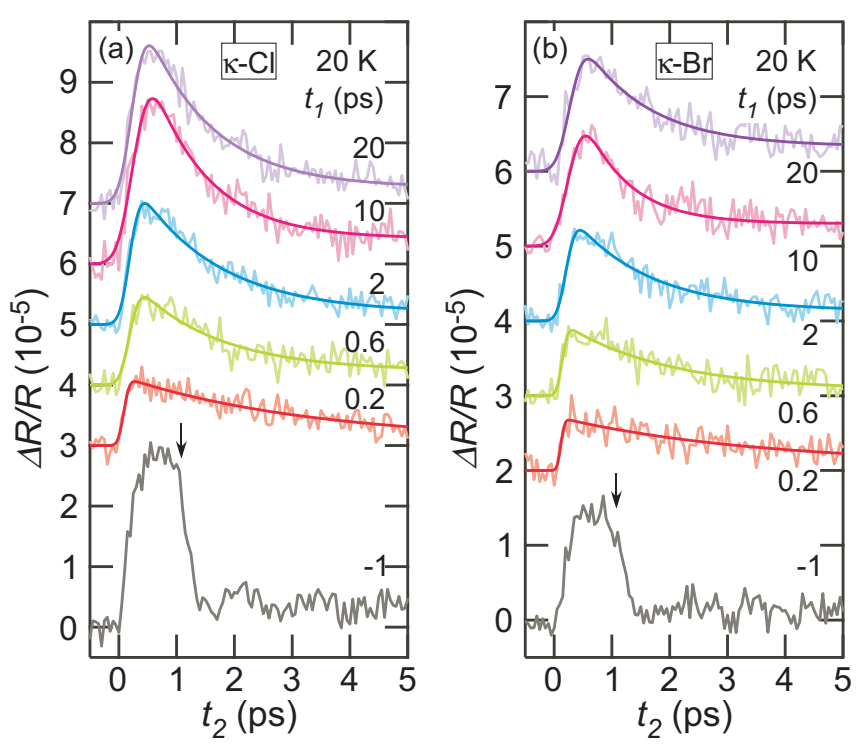

FIG. 2. Transient change in reflectivity $\Delta R / R$ as a function of $t_{2}$ at $T=20 \mathrm{~K}$ for $t_{1}=0.2,0.6,210$ and $20 \mathrm{ps}$ in (a) $\kappa-\mathrm{Cl}$ and (b) $\kappa-\mathrm{Br}$. The measurements were performed with fluence of the $\mathrm{P}$ pulse of $F=231 \mu \mathrm{J} / \mathrm{cm}^{2}$. The solid lines are the result fitted by Eq. (2). The data are shifted for clarity. The arrows denote arrival of the $\mathrm{P}$ pulse.

in Fig. 1(c). These three beams were almost perpendicular to the sample surface. The transient signal was detected by modulating only the intensity of the pump beam using a lockin amplifier synchronized to the optical chopper. Therefore, the contribution of an unchopped P-pulse beam to the transient reflectivity, such as coherent artifacts, was eliminated. Generally, in a gapped state the energy gap is suppressed by the P-pulse irradiation and gradually recovers again with time. By controlling the delay $t_{1}$ and conducting pump probe measurements, we can investigate the recovery of the gapped state, which can be probed by means of the carrier relaxation dynamics.

\section{RESULTS}

Figure 2(a) shows transient changes in reflectivity $\Delta R / R$ for different $t_{1}$ (the delay between $\mathrm{P}$ and pump pulses) in $\kappa-\mathrm{Cl}$ at $T=20 \mathrm{~K}$. Here $\Delta R / R$ reflects carrier dynamics in the Mott state characterized by a single exponential decay. When the $\mathrm{P}$ pulse arrives after the pump pulse $\left(t_{1}=-1 \mathrm{ps}\right), \Delta R / R$ shows a sudden decrease at around $t_{2}=1 \mathrm{ps}$ (indicated by the arrow), suggesting a quench of the Mott state upon arrival of the $\mathrm{P}$ pulse. When the P pulse arrives just before the pump pulse, at $t_{1}=0.2 \mathrm{ps}$, the signal amplitude, corresponding to the gap-induced bottleneck dynamics, becomes smaller than that at $t_{1}<0$. This indicates that the Mott state is suppressed on an $\sim 100$ fs timescale after irradiation by the P pulse. As $t_{1}$ increases, the signal amplitude gradually increases, suggesting that the Mott state emerges again. In $\kappa$ - $\mathrm{Br}$ a similar behavior is observed, as shown in Fig. 2(b).

In order to investigate time evolution of the signal amplitude $A_{1,2}$ and decay time $\tau$, we fit the transient reflectivity data

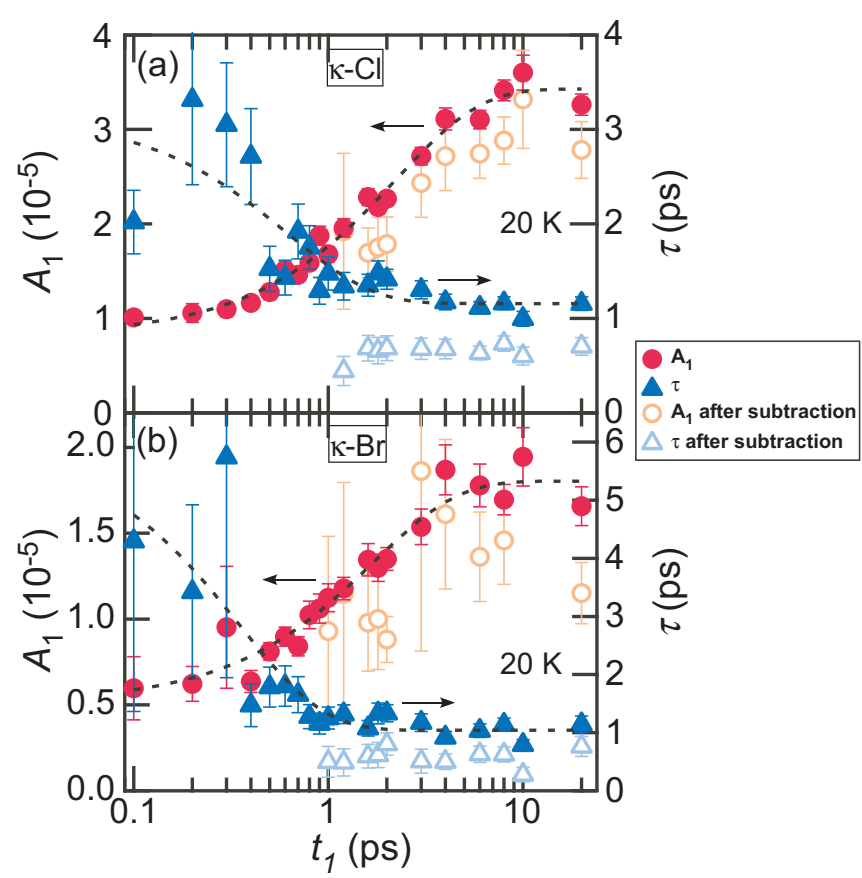

FIG. 3. $t_{1}$ dependences of the amplitude $A_{1}$ and decay time $\tau$ at $T=20 \mathrm{~K}$ in (a) $\kappa-\mathrm{Cl}$ and (b) $\kappa-\mathrm{Br}$, for $F=231 \mu \mathrm{J} / \mathrm{cm}^{2}$. The dashed lines are the results obtained by fitting with the single exponential function. As a result of the fit, the recovery time of $A_{1}$ is $\sim 2.2 \mathrm{ps}$ for $\kappa-\mathrm{Cl}$ and $\sim 1.7 \mathrm{ps}$ for $\kappa-\mathrm{Br}$.

by [27]

$$
\begin{aligned}
\frac{\Delta R}{R}\left(t_{2}\right)= & \frac{A_{1}}{2} \exp \left(-\frac{t_{2}-t_{0}}{\tau}\right) \operatorname{erfc}\left(\frac{\tau_{q}^{2}-4\left(t_{2}-t_{0}\right) \tau}{2 \sqrt{2} \tau_{q} \tau}\right) \\
& +\frac{A_{2}}{2} \operatorname{erfc}\left(-\frac{\sqrt{2}\left(t_{2}-t_{0}\right)}{\tau_{q}}\right)
\end{aligned}
$$

where $t_{0}$ and $\tau_{q}$ correspond to the time at which the pump pulse arrives and the pulse width described by a Gaussian function, respectively. $t_{0}$ and $\tau_{q}$ were also used as free parameters during the fitting. For component $A_{2}$, the relaxation time is assumed to be infinite.

In Fig. 3(a), the amplitude $A_{1}$ (solid circles) and $\tau$ (solid triangles) are plot on the left and right axes, respectively, for $\kappa-\mathrm{Cl}$. We note that the $A_{2}$ values are one order of magnitude lower than those of $A_{1}$ and independent of $t_{1}$. As $t_{1}$ increases, $A_{1}$ increases gradually, corresponding to recovery of the Mott state. On the other hand, $\tau$ is estimated to be $\sim 3$ ps below $t_{1} \sim 0.5 \mathrm{ps}$ and seems to drop steeper than $A_{1}$ rises with increasing $t_{1}$.

However, the different time evolutions of $A_{1}$ and $\tau$ are inconsistent with the assumption of a single relaxingcomponent transient reflectivity. If $A_{1}$ is considered a single component, the dynamics with a long decay time of $\sim 3 \mathrm{ps}$ changes to that with a short decay time of $\sim 1 \mathrm{ps}$ at $t_{1} \sim 0.5$ ps. For $t_{1}<0.5 \mathrm{ps}$, an anomaly due to such a change in dynamics is expected to appear in the transient reflectivity at $t_{2}=0.5 \mathrm{ps}-t_{1}$, but no anomalies are observed (see the transient response at $t_{1}=0.2 \mathrm{ps}$ ). The transient response must therefore be composed of at least two relaxing components: 

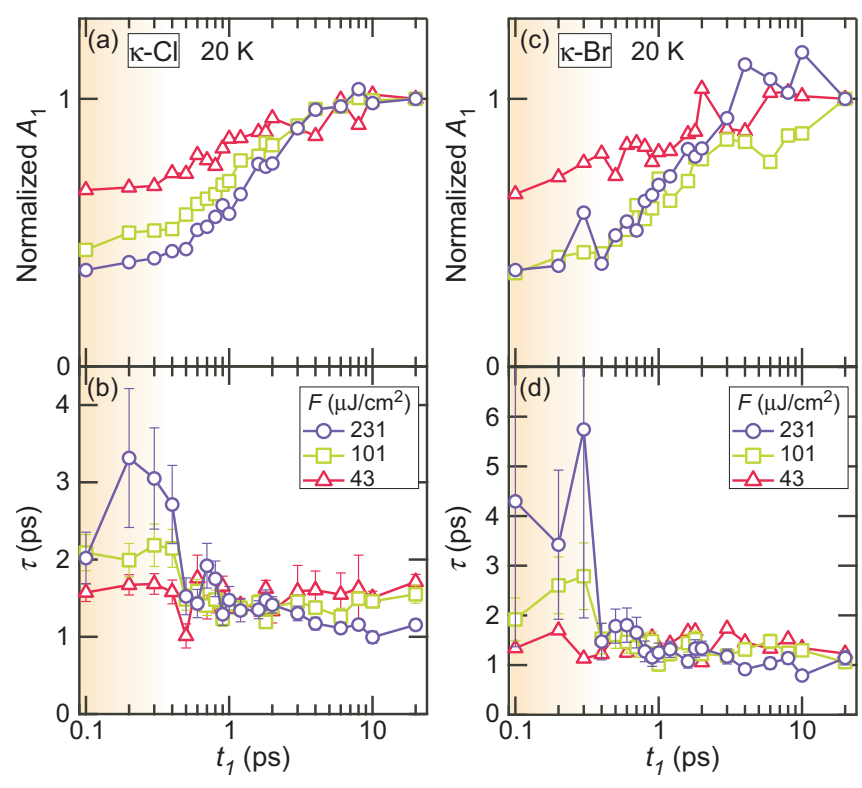

FIG. 4. (a) Normalized $A_{1}$ and (b) decay time $\tau$ as a function of $t_{1}$ at $T=20 \mathrm{~K}$ for $F=43,101$, and $231 \mu \mathrm{J} / \mathrm{cm}^{2}$ in $\kappa-\mathrm{Cl}$. (c) Normalized $A_{1}$ and (d) decay time $\tau$ as a function of $t_{1}$ at $T=$ $20 \mathrm{~K}$ for $F=43,101$, and $231 \mu \mathrm{J} / \mathrm{cm}^{2}$ in $\kappa-\mathrm{Br}$.

a slow one, which is independent of $t_{1}$, with a decay time of $\sim 3$ ps and a fast one, which grows with increasing $t_{1}$, with a decay time of $\sim 1 \mathrm{ps}$.

To verify the above hypothesis, $A_{1}$ and $\tau$ of the fast component were obtained from the data with the transient reflectivity at $t_{1}=0.2 \mathrm{ps}$, where the slow component is dominant, subtracted. In Figs. 3(a) and 3(b), the $t_{1}$ dependences of the fast component $A_{1}$ (open circles) and $\tau$ (open triangles) obtained upon subtraction are also presented. In both salts, the time evolutions of $A_{1}$ obtained from the subtracted data are beyond $t_{1} \sim 1 \mathrm{ps}$, similar to those obtained from the unsubtracted transient data by the single fast component analysis, albeit with slightly different values of $A_{1}$ and $\tau$.

Irrespective of the subtraction, we observe $t_{1}$-independent $\tau$ and $A_{1}$ recovering on an $\sim 2 \mathrm{ps} t_{1}$ timescale, which was obtained by fitting with the single exponential function as shown in Fig. 3. We associate the suppression of the fast decay component to suppression of the Mott state and the associated single-particle dynamics [19]. The long-lived component is not mentioned further in this paper because there are some unknowns and their origin would be out of context.

To confirm whether the gapped state was fully suppressed and the $t_{1}$ independence of $\tau$ is observed systematically, we measured the P-pulse fluence dependence of the recovery dynamics of the Mott state. Figures 4(a) and 4(b) show the normalized $A_{1}$ and $\tau$ as a function of $t_{1}$ for $F=43,101$, and $231 \mu \mathrm{J} / \mathrm{cm}^{2}$ for $\kappa-\mathrm{Cl}$. Since the subtraction of the slowly relaxing background component is not possible at low $F$, we use the single relaxing component analysis without the subtraction. As the $\mathrm{P}$ fluence decreases, the normalized $A_{1}$ at short $t_{1}$ becomes less suppressed.

In the previous measurements, with the excitation pulses at either 0.95 or $3.1 \mathrm{eV}[17,19]$, the amplitude of the transient reflectivity deviated from the linear variation at
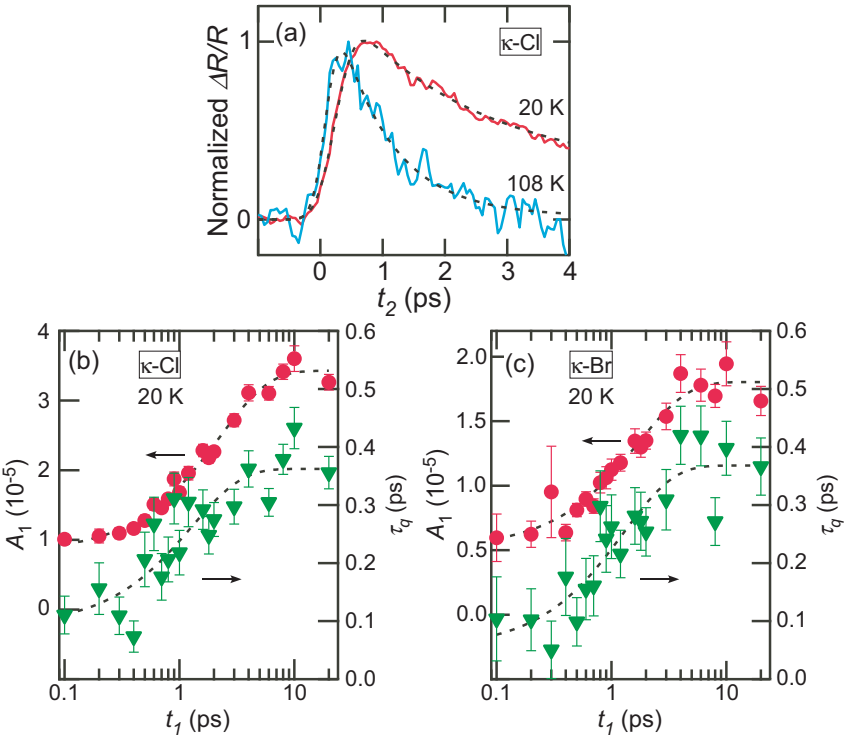

FIG. 5. (a) Comparison of the polarized pump probe reflectivity transients [17] $\Delta R / R$ in $\kappa-\mathrm{Cl}$ at $T=20 \mathrm{~K}$ and $108 \mathrm{~K}$. $t_{1}$ dependences of $A_{1}$ and $\tau_{q}$ for $F=231 \mu \mathrm{J} / \mathrm{cm}^{2}$ in (b) $\kappa-\mathrm{Cl}$ and (c) $\kappa-\mathrm{Br}$.

$F_{\mathrm{c}} \sim 100 \mu \mathrm{J} / \mathrm{cm}^{2}$, indicating the onset of partial suppression of the Mott state above $F_{\mathrm{c}}$. In the present case the increase of $A_{1}$ at the initial stage of the recovery with decreasing $\mathrm{P}$ fluence also indicates that the suppression of the Mott is incomplete at lower P-pulse fluences.

The short- $t_{1}$ decrease of $\tau$ with the decreasing P-pulse fluence [Fig 4 (b)], however, is an artifact of the single relaxation component analysis since the increase of $A_{1}$ in the early stages obscures the weaker slow transient reflectivity component and leads to an apparent change in $\tau$. At the lowest P-pulse fluence the short- $t_{1} \tau$ converges towards the long- $t_{1}$ value. The true fast component $\tau$ can therefore be considered virtually $t_{1}$ independent at any P-pulse fluence, while it slightly increases with decreasing P-pulse fluence. Since $\tau$ increases with decreasing temperature [19], we attribute this to a smaller heat buildup at lower P-pulse fluences.

In $\kappa$ - $\mathrm{Br}$, similar fluence dependences of the normalized $A_{1}$ and $\tau$ are observed, as shown in Figs. 4(c) and 4(d), respectively.

To illuminate further the recovery of the Mott state from another point of view, we focus on the $t_{1}$ dependence of $\tau_{q}$ that corresponds to the rise time of the transient reflectivity. Figure 5(a) shows the normalized transient reflectivity in $\kappa-\mathrm{Cl}$ obtained from the previous pump probe spectroscopy data [17]. The initial rise of the transient reflectivity in the gapped Mott state at $20 \mathrm{~K}$ is slower than that of the metallic response at $108 \mathrm{~K}$. The value of $\tau_{q}$ is estimated to be $0.48 \mathrm{ps}$ at $20 \mathrm{~K}$ and $0.33 \mathrm{ps}$ at $108 \mathrm{~K}$, indicating that the slow rise is a feature of the gapped state.

Figures 5(b) and 5(c) show $t_{1}$ dependences of $\tau_{q}$ (right axis) together with that of $A_{1}$ (left axis) in $\kappa-\mathrm{Cl}$ and $\kappa-\mathrm{Br}$, respectively. As $t_{1}$ increases, $\tau_{q}$ increases from $\sim 0.1$ to $\sim 0.4$ ps. The $t_{1}$ dependence appears to be quite similar to that of $A_{1}$ in both salts. This finding supports the interpretation that the PG is suppressed immediately after the P-pulse irradiation and reemerges again with increasing $t_{1}$. 


\section{DISCUSSION}

Like in the cases of charge density waves [6] and superconductors $[7,8,28]$, we associate the suppression of the ultrafast transient reflectivity after a strong ultrafast-optical-pulse excitation to the suppression of the gap in the electronic density of states. In the $\kappa-\mathrm{Cl}$ salt the gap is associated with the bulk Mott insulating state, while in the $\kappa$-Br salt the presence of the PG was attributed to the photoinduced phase separation into metallic and Mott insulating phases that is, presumably [19], a consequence of photoexcitation-induced terminal ethylene group disorder leading to an effective negative pressure effect and a local modulation of $t / U$.

Our experiments are stroboscopic with a repetition rate of $100 \mathrm{kHz}$, and such phase separation has been observed also for relatively slow quenches [29]. The photoexcited phase separation is therefore most likely a buildup effect of repetitive quenches of the electronic system, and the structural phase separation can be considered rather static on the picosecond timescale of a single three-pulse sequence, as indicated by the rather similar ultrafast transient reflectivity [19] in both salts. The present results confirm such a picture with very similar behavior in both salts, where in the $\kappa$ - $\mathrm{Br}$ salt the observed transient reflectivity dynamics originates from the phase-separated Mott insulating phase. In the following we will therefore discuss the data in both salts on equal footing.

Our data indicate that the Mott insulating state and the associated gap-induced bottleneck are suppressed on an $\sim 100 \mathrm{fs}$ timescale in both salts, while the gradual increase of the fast component amplitude with increasing $t_{1}$ on a few-picosecond timescale corresponds to the recovery of the Mott insulating state. Strikingly, $\tau$ of the fast component is independent of $t_{1}$. Such a difference in the recovery dynamics for the amplitude and relaxation time is usually not observed in the cooperatively ordered electronic states characterized by spontaneous symmetry breaking, such as charge-density-wave [6] and superconducting states $[7,8]$, and was observed only in the PG state in $\mathrm{Bi}_{2} \mathrm{Sr}_{2} \mathrm{CaCu}_{2} \mathrm{O}_{8+x}$ (Bi2212) [28].

In the cooperatively ordered systems $\tau$ shows a steep increase with decreasing $t_{1}$. The behavior reflects the critical slowing down of the order parameter fluctuations in the vicinity of a symmetry-breaking phase transition. In the initial stage of the recovery, $\tau$ is linked to the energy gap $\Delta_{\text {gap }}$ and described as $\tau \sim 1 / \Delta_{\text {gap }}$ because a relaxation bottleneck effect is dominant [25]. Therefore, the recovery times of the amplitude and decay time have similar values [6,7].

In the case of the PG-related dynamics in Bi2212 [28], on the other hand, $\tau$ is constant, while the signal amplitude recovers with increasing $t_{1}$. This is because the PG state is considered to arise from the carrier localization due to Coulomb interaction and lattice strain, indicating that the PG formation is not cooperative. In other words, PG is filled by the appearance of in-gap states rather than closed after the P-pulse irradiation. In this case, the intense pulse irradiation is considered to lead to depletion of the lower band, showing the saturation behavior observed at high fluences.

Our results are similar to the PG recovery dynamics in Bi2212. In the following, we discuss the possible mechanisms. In the Mott insulator, carriers are localized at each lattice site by the strong Coulomb repulsion, resulting in an energy gap $\sim U$ for the charge excitation. In the present charge transfer salts the lattice sites correspond to dimers. As a result there are two orbitals at each lattice site, and the relevant electronic structure includes an additional band in the proximity of the Hubbard split half-filled band. Optical spectroscopy [15] indicates the transition between the split Hubbard subbands at $\sim 0.3 \mathrm{eV}$ and another transition at $\sim 0.4 \mathrm{eV}$, corresponding to the intradimer transition.

Theoretically, the Mott gap seems to be quite robust with respect to the charge-neutral photoexcitation [30,31]. In the present case, however, there is a significant particle-hole asymmetry due to the presence of the additional band. Strong photoexcitation therefore results in an effective chemical potential shift that is akin to transient photodoping.

Recent theoretical studies suggested that a tiny carrier doping induces electronic states in the lower Hubbard band (LHB) [32,33]. The Mott gap is also quite robust with respect to doping and does not close at low doping. However, the Fermi energy is pushed into the LHB, resulting in a gapless metallic state with no bottleneck for photoexcited carrier relaxation. Moreover, in the presence of an additional band photoexcitation and/or a chemical potential, the shift can lead to a first-order phase transition into a metastable metallic state with a complete collapse of the Mott gap, as suggested [34] in the case of $\mathrm{V}_{2} \mathrm{O}_{3}$. Either scenario of the gap filling [33] or first-order phase transition with Mott gap collapse [34] would lead to the absence of criticality and, as a result, $\tau$ that is independent of $t_{1}$.

In our experiments, the carriers excited to the high-energy states relax on an $\sim 100 \mathrm{fs}$ timescale to the above-mentioned bands. The P-pulse irradiation therefore leads to the ultrafast doping and a change in the electronic band structure. In order to determine which scenario is appropriate, it is useful to estimate the photoexcited carrier density $\delta$. Assuming that each photon excites one or more carriers due to the avalanche multiplication carriers, $\delta$ can be defined as the following form:

$$
\delta \gtrsim \frac{F V_{\mathrm{dim}}}{l_{\mathrm{opt}} \hbar \omega},
$$

where $V_{\mathrm{dim}}, l_{\mathrm{opt}}$, and $\hbar \omega$ are the volume of a dimer, optical penetration depth, and photon energy, respectively. For $F=$ $231 \mu \mathrm{J} / \mathrm{cm}^{2}$, using $V_{\text {dim }}=1650 \AA^{3}[35]$ and $l_{\text {opt }}=3 \mu \mathrm{m}$ for $\hbar \omega=1.53 \mathrm{eV}$, values assumed to be similar to those of $\kappa$-(BEDT-TTF $)_{2} \mathrm{Cu}$ (NCS $)_{2}$ [36], $\delta$ is calculated to be $\gtrsim 0.005$ per dimer. In this calculation, the reflection loss is not taken into account, so the actual minimum value of $\delta$ would be smaller than 0.005 . On the other hand, the number of carriers excited by a single photon could be larger due to the avalanche multiplication during the hot carrier relaxation. At weak excitations, when the gapped state is not suppressed, the maximum possible multiplication factor is $\hbar \omega / \Delta_{\text {gap }} \sim 15$, with $\Delta_{\text {gap }} \sim$ $0.1 \mathrm{eV}$ [14-16], assuming no energy dissipation by the lattice degrees of freedom. At high excitations, when the gapped state is suppressed, such an estimate is not possible without a detailed model. Nevertheless, based on the low excitation density, it is unlikely that $\delta$ would significantly exceed a magnitude of a few percent.

The first-order phase transition with the Mott gap collapse requires photoexcited carrier densities in the range of 0.15 to 0.2 [34], which is significantly larger than the above estimate. 
The gap-filling scenario [33], on the other hand, requires only a small carrier doping, which is more consistent with our estimation. We therefore propose that the ultrafast Mott state suppression in the $\kappa-\mathrm{Cl}$ and $\kappa-\mathrm{Br}$ salts is due to the MottHubbard gap filling rather than the first-order phase transition with collapse of the gap.

The rather fast Mott-state recovery timescale of $\sim 2 \mathrm{ps}$ is then set by the excess energy transfer to the lattice degrees of freedom since any electronic relaxation is expected to be faster due to the absence of any gap at the transiently shifted chemical potential.

As referred to earlier, in $\kappa-\mathrm{Br}$, the transient reflectivity dynamics is attributed to the phase-separated Mott insulating phase. In this case, we can interpret the data as follows. Just after the irradiation of intense $P$ pulses, the excited states are limited, indicating that the pump pulse no longer generates a Mott insulating state. As $t_{1}$ increases, excitation by the pump pulse becomes possible, leading to the recovery of the dynamics amplitude. During the recovery, the pump pulse causes the photocarrier doping effect to occur simultaneously in the pump-induced Mott gap, showing no change in the relaxation time. This is the reason why the recovery dynamics of $\kappa$ - $\mathrm{Br}$ is very similar to that of $\kappa$-Cl.

\section{CONCLUSIONS}

By applying the three-pulse optical measurement technique to the strongly correlated organic conductors $\kappa-\mathrm{Cl}$ and $\kappa-\mathrm{Br}$, we observed the dynamics of the photoinduced Mottstate quench and recovery under nonequilibrium conditions. In both salts the Mott state is suppressed by the intense $\mathrm{P}$ pulse on a 100 fs timescale and recovers on a few-picosecond timescale. The difference in the dynamics for the transient reflectivity amplitude and relaxation time, which are observed in both salts, suggests that the unique properties of the Mott state, such as the appearance of electronic states at the Fermi energy upon carrier doping, play an important role in the Mott-state quench and recovery.

\section{ACKNOWLEDGMENTS}

The authors would like to thank D. Svetin, A. Mraz, J. Ravnik, V. Nasretdinova, Y. Gerasimenko, I. Vaskivskyi, and M. Naseska for experimental support. S.T. would like to thank the Faculty of Engineering, Hokkaido University, for partial financial support. Y.T. acknowledges the support of the JSPS KAKENHI (Grant No. JP19H05826).
[1] M. Imada, A. Fujimori, and Y. Tokura, Rev. Mod. Phys. 70, 1039 (1998).

[2] P. A. Lee, N. Nagaosa, and X.-G. Wen, Rev. Mod. Phys. 78, 17 (2006).

[3] K. Kanoda, Hyperfine Interact. 104, 235 (1997).

[4] Y. Kawasugi, H. M. Yamamoto, N. Tajima, T. Fukunaga, K. Tsukagoshi, and R. Kato, Phys. Rev. B 84, 125129 (2011).

[5] H. M. Yamamoto, M. Nakano, M. Suda, Y. Iwasa, M. Kawasaki, and R. Kato, Nat. Commun. 4, 2379 (2013).

[6] R. Yusupov, T. Mertelj, V. V. Kabanov, S. Brazovskii, P. Kusar, J. H. Chu, I. R. Fisher, and D. Mihailovic, Nat. Phys. 6, 681 (2010)

[7] I. Madan, P. Kusar, V. V. Baranov, M. Lu-Dac, V. V. Kabanov, T. Mertelj, and D. Mihailovic, Phys. Rev. B 93, 224520 (2016).

[8] I. Madan, V. V. Baranov, Y. Toda, M. Oda, T. Kurosawa, V. V. Kabanov, T. Mertelj, and D. Mihailovic, Phys. Rev. B 96, 184522 (2017).

[9] M. Naseska, A. Pogrebna, G. Cao, Z. A. Xu, D. Mihailovic, and T. Mertelj, Phys. Rev. B 98, 035148 (2018).

[10] T. Mertelj, P. Kusar, V. V. Kabanov, P. Giraldo-Gallo, I. R. Fisher, and D. Mihailovic, Phys. Rev. Lett. 110, 156401 (2013).

[11] W. Zurek, Phys. Rep. 276, 177 (1996).

[12] T. W. B. Kibble and G. E. Volovik, JETP Lett. 65, 102 (1997).

[13] B. J. Powell and R. H. McKenzie, J. Phys.: Condens. Matter 18, R827 (2006).

[14] K. Kornelsen, J. E. Eldridge, H. H. Wang, H. A. Charlier, and J. M. Williams, Solid State Commun. 81, 343 (1992).

[15] D. Faltermeier, J. Barz, M. Dumm, M. Dressel, N. Drichko, B. Petrov, V. Semkin, R. Vlasova, C. Meziere, and P. Batail, Phys. Rev. B 76, 165113 (2007).

[16] M. Dumm, D. Faltermeier, N. Drichko, M. Dressel, C. Meziere, and P. Batail, Phys. Rev. B 79, 195106 (2009).

[17] S. Tsuchiya, K. Nakagawa, H. Taniguchi, and Y. Toda, J. Phys. Soc. Jpn. 88, 074706 (2019).
[18] S. Tsuchiya, K. Nakagawa, J. Yamada, H. Taniguchi, and Y. Toda, Phys. Rev. B 96, 134311 (2017).

[19] Y. Toda, T. Mertelj, T. Naito, and D. Mihailovic, Phys. Rev. Lett. 107, 227002 (2011).

[20] H. Anzai, J. M. Delrieu, S. Takasaki, S. Nakajima, and J. Yamada, J. Cryst. Growth 154, 145 (1995).

[21] J. Williams, A. M. Kini, H. H. Wang, K. D. Carlson, U. Geiser, L. K. Montgomery, G. J. Pryka, D. M. Watkins, W. K. Kwok, J. E. Schirber, D. L. Overmyer, D. Jung, and H. M. Whnagbo, Inorg. Chem. 29, 3272 (1990).

[22] W. Y. Ching, Y.-N. Xu, Y. C. Jean, and Y. Lou, Phys. Rev. B 55 2780 (1997)

[23] H. Akutsu, K. Saito, and M. Sorai, Phys. Rev. B 61, 4346 (2000)

[24] P. B. Allen, Phys. Rev. Lett. 59, 1460 (1987).

[25] A. Rothwarf and B. N. Taylor, Phys. Rev. Lett. 19, 27 (1967).

[26] V. V. Kabanov, J. Demsar, B. Podobnik, and D. Mihailovic, Phys. Rev. B 59, 1497 (1999).

[27] T. Mertelj, L. Stojchevska, N. D. Zhigadlo, J. Karpinski, and D. Mihailovic, Phys. Rev. B 87, 174525 (2013).

[28] I. Madan, T. Kurosawa, Y. Toda, M. Oda, T. Mertelj, and D. Mihailovic, Nat. Commun. 6, 6958 (2015).

[29] O. J. Taylor, A. Carrington, and J. A. Schlueter, Phys. Rev. B 77, 060503(R) (2008).

[30] M. Eckstein and P. Werner, Sci. Rep. 6, 21235 (2016).

[31] M. Eckstein and P. Werner, Phys. Rev. B 84, 035122 (2011)

[32] M. Kohno, Phys. Rev. Lett. 105, 106402 (2010).

[33] M. Kohno, Phys. Rev. Lett. 108, 076401 (2012).

[34] M. Sandri and M. Fabrizio, Phys. Rev. B 91, 115102 (2015).

[35] U. Geiser, A. J. Schultz, H. H. Wang, D. M. Watkins, D. L. Stupka, and J. M. Williams, Phys. C (Amsterdam, Neth.) 174, 475 (1991)

[36] A. Ugawa, G. Ojima, K. Yakushi, and H. Kuroda, Phys. Rev. B 38, 5122(R) (1988). 